\title{
Fiber Optic Biosniffer (Biochemical Gas Sensor) for Gaseous Dimethyl Sulfide
}

\author{
Hirokazu Saito, Yuki Hashimoto, ${ }^{1}$ Takeshi Minamide, ${ }^{1}$ Takuo Kon, ${ }^{1}$ \\ Koji Toma, Takahiro Arakawa, and Kohji Mitsubayashi* \\ Department of Biomedical Devices and Instrumentation, Institute of Biomaterials and Bioengineering, \\ Tokyo Medical and Dental University, 2-3-10 Kanda-Surugadai, Chiyoda-ku, Tokyo 101-0062, Japan \\ ${ }^{1}$ Course of Electrical Engineering, Graduate School of Engineering, Tokai University, \\ 1117 Kitakaname, Hiratsuka, Kanagawa 259-1292, Japan
}

(Received March 14, 2016; accepted July 13, 2016)

Keywords: optical biosniffer, oxygen-sensitive optical fiber, DMS vapor, flavin-containing monooxygenase

An optical biosniffer for dimethyl sulfide (DMS; seaweed-odor substance) was constructed by immobilizing flavin-containing monooxygenase type 3 (FMO3) onto the tip of a fiber optic oxygen sensor with an oxygen-sensitive ruthenium organic complex (excitation: $470 \mathrm{~nm}$, fluorescence: $600 \mathrm{~nm}$ ) with a tube ring. A reaction unit for circulating buffer solution was applied to the tip of the sensor device. After the experiment in the liquid phase, the sniffer device was applied to gas analysis using a gas flow measurement system with a gas generator. The optical device could be applied to detect the oxygen consumption induced by the FMO3 enzymatic reaction with DMS application. The sensor in the liquid phase was used to measure DMS solution from 0.52 to 7.32 $\mathrm{mmol} / \mathrm{l}$, with a cyclic reaction with ascorbic acid (AsA) as the reducing reagent. Then, the sniffer device with the reaction unit was calibrated against DMS vapor from 2.1-126 ppm with good gas selectivity based on the FMO3 substrate specificity. The device could be used to monitor the concentration change of gaseous DMS using the reaction unit, in which the substrate and product substances flush out at the sensor tip and the enzyme membrane.

\section{Introduction}

Dimethyl sulfide (DMS, seaweed-odor substance) is a colorless solution in the liquid phase and one of the volatile sulfur compounds with characteristic malodor in the gas phase as defined by the International Occupational Safety and Health Information Center. ${ }^{(1)}$ The substance decomposes upon burning, producing toxic and corrosive fumes (sulfur oxides), and reacts violently with oxidants causing fire and explosion hazard. The flash point, auto ignition temperature, and explosive limits of DMS are $-49^{\circ} \mathrm{C}, 205^{\circ} \mathrm{C}$, and $2.2-19.7 \mathrm{vol} \%$ in air, respectively, as defined by International Chemical Safety Cards (ICSC). ${ }^{(1)}$

A harmful contamination of the air can occur rather quickly by the evaporation of DMS at $20{ }^{\circ} \mathrm{C}$. The substance irritates the eyes and the skin. In the human body, flavin-containing monooxygenase (FMO, EC 1.14.13.8) as one of the xenobiotic-metabolizing enzymes has been reported to catalyze the oxidation of sulfuric and nitric compounds including DMS. ${ }^{(2)}$ FMO type

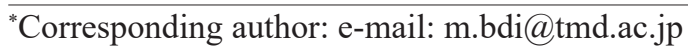


3 (FMO3) can be expressed from human FMO3 cDNA using a baculovirus expression system ${ }^{(3,4)}$ and commercialized. Oxygen consumption by the enzyme reaction has been used for analyzing the enzyme activity or the substrate concentration including DMS.(4) On the other hand, some optical fibers with chemical sensitivity have been commercialized..$^{(5-7)}$ An oxygen-sensitive optical fiber coated with a ruthenium-organic complex reacts to the existence of oxygen molecule in both the liquid and gas phases. The optical fiber with biocatalyst has been expected to be applied to new sensing devices for biological analysis including gas analysis for volatile organic compounds (VOCs). We have also reported several types of optical biochemical sensor. ${ }^{(8-10)}$

In this work, we developed an optical biosniffer using FMO enzyme for the measurement of gaseous DMS. The performance of the sensor is evaluated with a gas flow measurement system for gas-phase detection.

\section{Experimental}

\subsection{Construction of optical biosniffer for dimethyl sulfide}

Figure 1 illustrates the structure of an optical biochemical gas sensor (the so-called "biosniffer") for DMS. The optical biosniffer consisted of an enzyme membrane, a reaction unit, and an oxygensensitive optical fiber \{[FOXY-R/RTV-Flat (silicone overcoat), Lot No. OX3200], 1/16" outer diameter, stainless-steel tube beveled, Ocean Optics, Inc., FL, USA $\}$. The optical fiber was coated

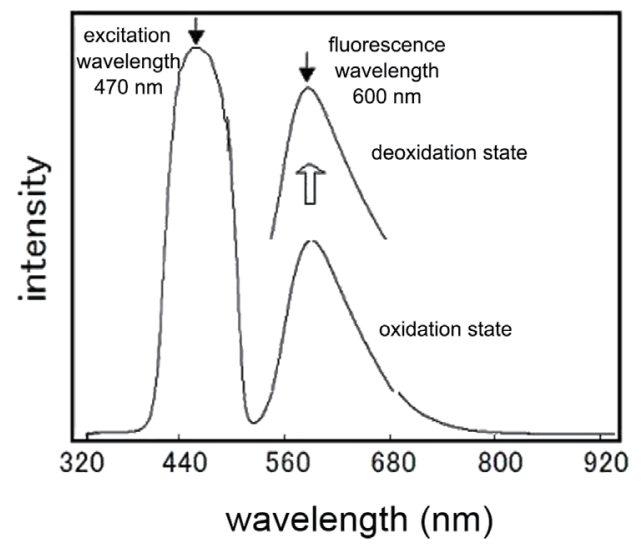

(a)
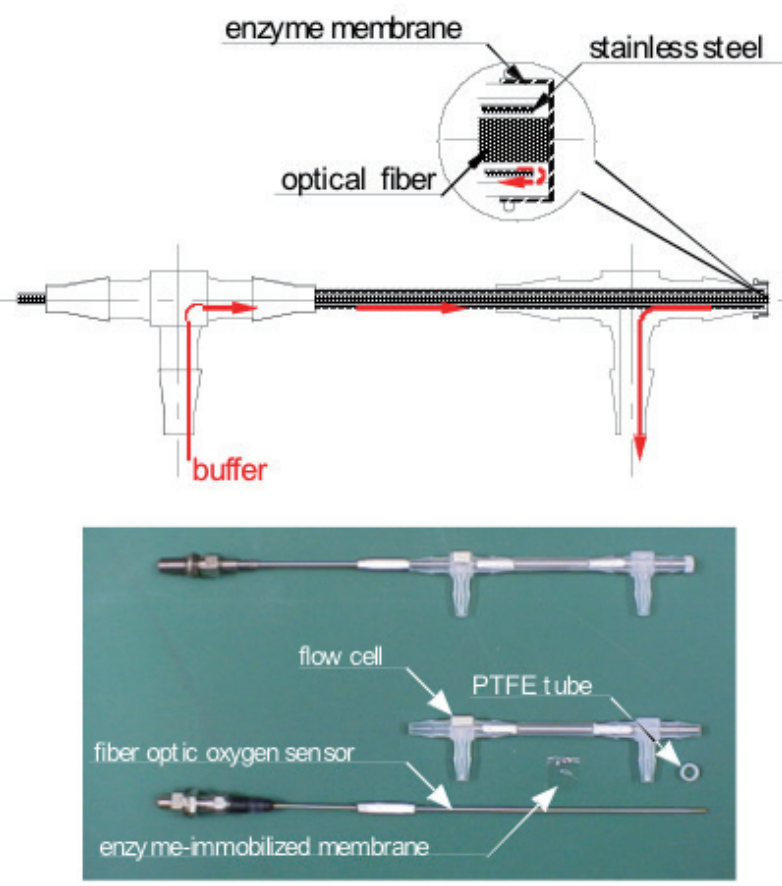

(b)

Fig. 1. (Color online) Structure and photograph (b) of optical biosniffer for DMS vapor, in which on FMOimmobilized membrane covered the sensor tip of an oxygen-sensitive optical fiber [its optical characteristics are illustrated in (a)]. 
by a sol-gel process with a ruthenium-organic fluorescent complex (excitation wavelength: 470 $\mathrm{nm}$, fluorescent wavelength: $600 \mathrm{~nm}$ ), which causes optical quenching in the presence of oxygen molecules in both the liquid and gas phases, as indicated in Fig. 1(a). FMO3 (EC 1.14.13.8, P233, $30200 \mathrm{pmol} / \mathrm{mg}$, from adult human liver; Gentest Corp., MA, USA) was used for constructing the optical biosniffer for DMS vapor. The enzyme is specific for catalyzing the nicotinamide adenine dinucleotide phosphate (NADPH)-dependent $N$-oxygenation of nucleophilic nitrogen-, sulfur-, and phosphorus-containing chemicals, drugs, and xenobiotics, including DMS as described above.

To immobilize the enzyme, the FMO3 solution was mixed with photo-cross-linkable polyvinyl alcohol containing stilbazolium groups (PVA[polyvinyl alcohol]-SbQ [stilbazole quaternized]; Grade: SPP-H-13 [bio]; degree of polymerization: 1700; SbQ content: 1.28 mol\%; Toyo Gosei Co., Ltd., Tokyo, Japan) in a volume ratio of $1: 2,{ }^{(11)}$ and the resultant mixture was placed onto a dialysis membrane (No. 157-0144-02, thickness: $15 \mu \mathrm{m}$, Technicon Chemicals Co., S.A., Orcq, Belgium), dried on a plastic plate and then spread over the surface of the membrane until it had permeated (observed as a darkening of the membrane). ${ }^{(8)}$ The dialysis membrane was placed in the dark below $10{ }^{\circ} \mathrm{C}$ for $1 \mathrm{~h}$ and then irradiated with fluorescent light for $30 \mathrm{~min}$ in order to photo-cross-link the PVA-SbQ solution, thus immobilizing the enzyme into the dialysis membrane. ${ }^{(11)}$ The FMO3 immobilized membrane was removed from the plastic plate and immersed in phosphate buffer $(\mathrm{pH} 8.0$, $100 \mathrm{mmol} / \mathrm{l}$ ) with $65 \mu \mathrm{mol} / \mathrm{l}$ of $\beta$-NADPH and $3.3 \mathrm{mmol} / 1$ of $\mathrm{MgCl}_{2}$. In order to prevent enzyme deactivation when not in use, the membrane was stored in a buffer below $10^{\circ} \mathrm{C}$.

The reaction unit was constructed by connecting T-tubes to each side of a stainless steel pipe [outer diameter (o.d.) $2.44 \mathrm{~mm}$, internal diameter (i.d.) $1.99 \mathrm{~mm}$ ] and the inner side edges of the two T-tubes were closed with sealing tape. The enzyme membrane was used to close one of the open edges of the reaction unit and fixed with a rubber O-ring [see the enlargement of Fig. 1(b)]. The fiber tip of the optical biosensor was inserted from another open edge to the reaction unit and adjusted so as to directly touch the surface of the enzyme membrane [see the enlargement of Fig. 1(b)]. Then the edge was also closed by the sealing tape. The buffer solution in the reaction unit flowed into the stainless-steel tube from the middle edge of the root-side T-tube to that of the tipside T-tube, thus rinsing and cleaning the fiber tip and enzyme membrane. The sensor tip was connected to the side hole of polytetrafluoroethylene (PTFE) tube (i.d. $6 \mathrm{~mm}$ ) that supplied the gaseous substances.

In order to amplify the output signal of the biodevices, the substrate regeneration cycle was applied for measuring DMS by coupling the monooxygenase with the reducing reagent system of ascorbic acid (AsA) (Fig. 2). ${ }^{(12-14)}$ The optical biosniffer would detect the oxygen consumption in the FMO3 enzymatic reaction with DMS.

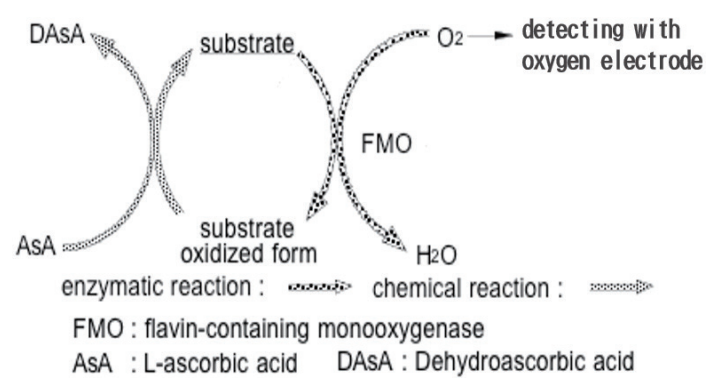

Fig. 2. Principle of cyclic reaction for signal-amplified biosniffer for DMS using FMO enzyme and AsA as reducing reagent. In this figure, DMS is represented as the substrate. 


\subsection{Evaluation of optical biosniffer for DMS}

The characteristics of the biosniffer for DMS vapor were evaluated using a batch-flow measurement system (Fig. 3). A gas generator (Permeater, type: PD-1B-2, Gastec Corp., Yokohama, Japan), which is a standard apparatus approved by the Ministry of Labor and the Ministry of the Environment in Japan and the Environmental Protection Agency (EPA) and the National Bureau of Standards (NBS) in the United States for gas calibration purposes, was used for supplying the required gaseous substances using standard air (approximately 20.9\% oxygen) purified using an activated-carbon filter.

A mass flow controller with a needle-valve regulator (RK1200, KOFLOC, Tokyo, Japan) was used to adjust the flow rate of the standard vapor, thus permitting the gaseous substance to flow the gas cell of the reaction unit with a final flow rate of $200 \mathrm{ml} / \mathrm{min}$.

Phosphate buffer solution ( $\mathrm{pH} 8.0,100 \mathrm{mmol} / \mathrm{l})$ with and without AsA in a carrier reservoir with the temperature maintained at $25{ }^{\circ} \mathrm{C}$ was circulated to the fiber tip and the enzyme membrane through the reaction unit of the bio-optical sniffer with a flow rate of $0.69 \mathrm{ml} / \mathrm{min}$ using a peristaltic pump (SJ-1211L, ATTO Corp., Tokyo, Japan).

A computer-controlled spectrophotometer (S2000, Ocean Optics, Inc., FL, USA) with an analogue-digital converter (DAQ700, PCMCIA A/D card with $100 \mathrm{kHz}$ sampling frequency, National Instruments Corporation, TX, USA) was optically connected to the biosniffer to monitor the optical quenching (fluorescence: $600 \mathrm{~nm}$ ) by oxygen consumption caused by the FMO catalytic reaction with DMS. The device output was monitored graphically on a continuous computer display and saved on a hard disk for later analysis. The gas selectivity of the optical biosniffer was evaluated using various odorous substances. In order to compare the sensor characteristics, different kinds of biosniffer were constructed using isomeric FMO enzyme (type 1: FMO1, type 5: FMO5).

A liquid-phase optical biosensor was also constructed using an immobilized enzyme membrane fabricated onto the fiber tip of the fiber oxygen-sensitive sensor with the rubber O-ring. The characteristics of the biosensor were also investigated using a batch measurement system.

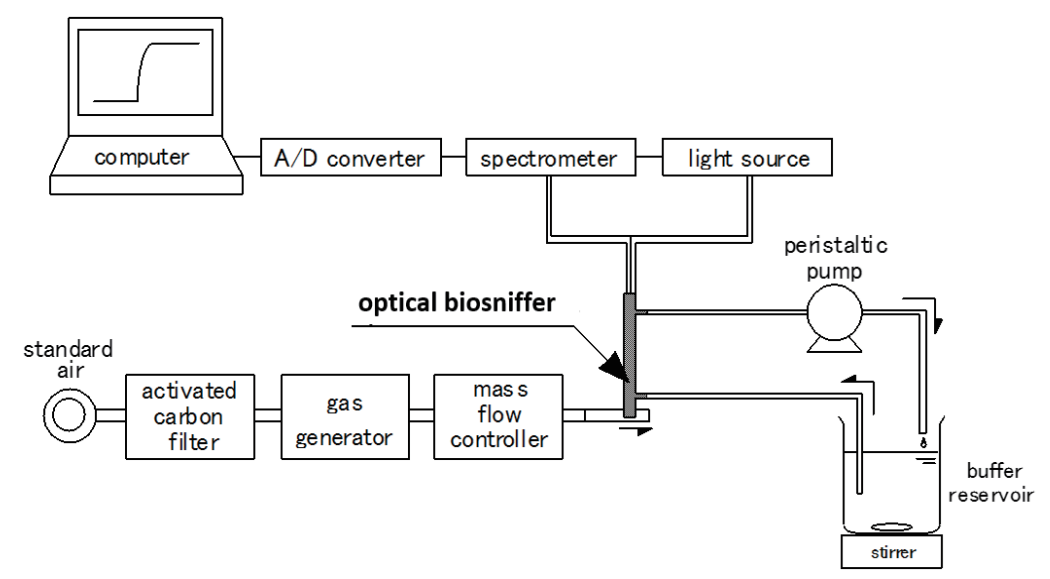

Fig. 3. Schematic diagram of gas flow measurement system for evaluating the characteristics of optical biosniffer for DMS in the gas phase. 


\section{Results and Discussion}

\subsection{Evaluation of optical biosensor for DMS in the liquid phase}

The optical biosensor for DMS in the liquid phase was evaluated prior to the biosniffer device in the gas phase. The calibration curves of the DMS biosensor with and without AsA $(10.0 \mathrm{mmol} /$ 1) in the liquid phase are shown in Fig. 4. In the figure, the sensor output is represented as a percentage of the steady-state current to the varying concentration of DMS for saturated output. As Fig. 4 indicates, both the sensor outputs without and with AsA in phosphate buffer were related to the concentration of DMS in the liquid phase over the ranges of $2.08-14.51$ and $0.52-7.32 \mathrm{mmol} /$ 1 , respectively, as deduced from exponential regression analysis of the log-log plots by the leastsquares method.

By comparing the two regression curves with and without AsA, the calibration range can be translated to a lower concentration level with a slight change in the slope of the regression curves by the addition of the reducing reagent, thus improving the lower detection limit from 2.08 to 0.52 $\mathrm{mmol} / \mathrm{l}$. The sensor output increases with increasing concentration of AsA as the reducing reagent, inducing the substrate regeneration cycle as described above. ${ }^{(9,10,12-14)}$ Since a substrate aggregation was markedly observed and there was little enhancement of the amplified output for the AsA concentration above $10.0 \mathrm{mmol} / \mathrm{l}$, the AsA concentration of $10.0 \mathrm{mmol} / \mathrm{l}$ in phosphate buffer was generally used in all experiments with the substrate regeneration cycle.

\subsection{Evaluation of optical biosniffer for DMS in the gas phase}

The calibration curve of the optical biosniffer for DMS in the gas phase is illustrated in Fig. 5. The optical sniffer was calibrated against gaseous DMS over the range of 2.1 to $126 \mathrm{ppm}$ (correlation coefficient of 0.994 ), deduced by exponential regression analysis of the log-log plot by the leastsquares method using the following equation:

$$
\text { Sensor output }(\text { counts })=31.4[\mathrm{DMS}(\mathrm{ppm})]^{0.55} \text {. }
$$

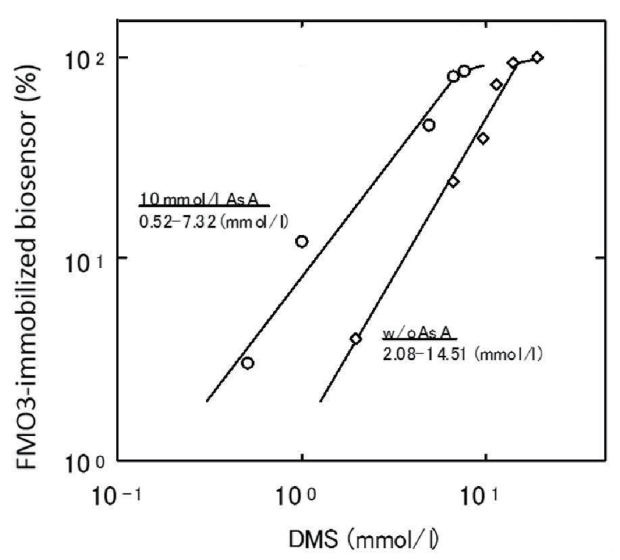

Fig. 4. Calibration curves of the FMO3-immobilized biosensor for DMS solution (open squares: w/o AsA, open circles: $10.0 \mathrm{mmol} / \mathrm{l} \mathrm{AsA}$ ).

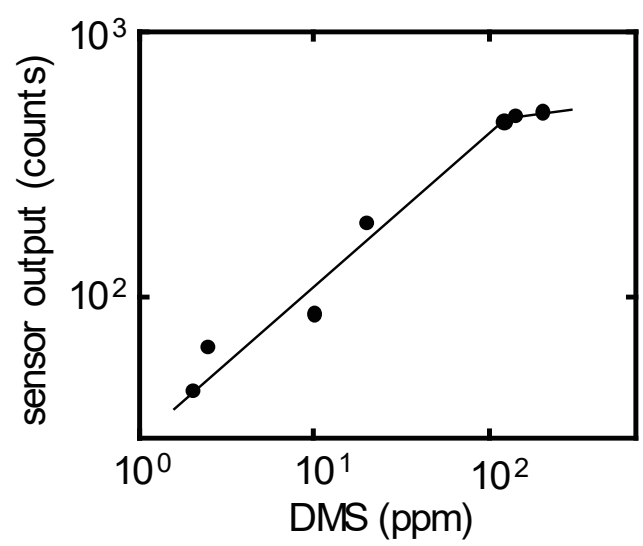

Fig. 5. Calibration curve of the optical biosniffer with $10.0 \mathrm{mmol} / 1 \mathrm{AsA}$ for DMS in the gas phase, covering the explosive-limit concentration (2.2 vol\%) defined by ICSC. 
The calibration range of the optical biosniffer for DMS vapor is lower than the explosive limits of DMS vapor (vol\% in air: 2.2-19.7), as described above.

In addition, the optical biosniffer was applied for a gas assessment of a seaweed sample as its food application. The sample was prepared by immersing $1 \mathrm{~g}$ of the dried seaweed with $5 \mathrm{ml}$ of distilled water, and stored in a container box $(790 \mathrm{ml})$ at $25{ }^{\circ} \mathrm{C}$ in order to generate the seaweed odor, as shown as Fig. 6. As a result of applying the resultant gas in the container, the optical sniffer successfully detected gaseous DMS. The concentration of DMS in the gas phase was calculated to be $7.0 \mathrm{ppm}$ in the container, which is consistent with the DMS concentration obtained using a commercially available detection tube.

The sniffer device with FMO3 gave a negligible response to most gaseous substances, whereas the application of trimethylamine (TMA) and methyl mercaptan (MM) induced an increase in the sensor output because FMO3 from human liver is a member of a polymorphic family of FMOs catalyzed in the oxidation of heteroatom-containing compounds in xenobiotic metabolism.

Figure 7 shows the radar chart of the optical biosniffer with FMO1, 3, and 5 for gaseous DMS of 35 and $70 \mathrm{ppm}$. The output values in the chart are nondimensionalized into a percentage of each device output relative to the maximum response signal of FMO3. Each triangle configuration was drawn using the outputs of the biosniffer device with FMO1, 3, and 5 for each gas analysis of DMS.
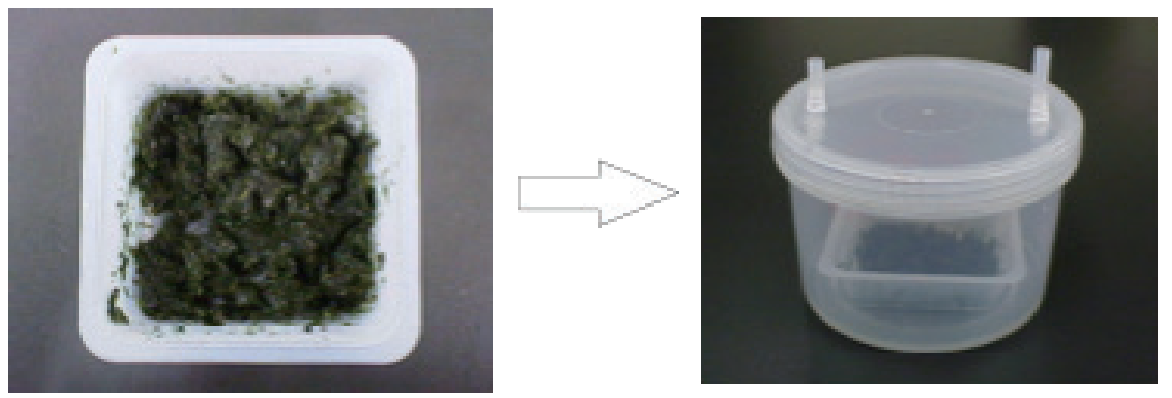

Fig. 6. (Color online) Seaweed sample ( $1 \mathrm{~g}$ with $5 \mathrm{ml}$ of distilled water) in a container box (790 $\mathrm{ml})$. The concentration of DMS in the gas phase was calculated to be $7.0 \mathrm{ppm}$ in the container.

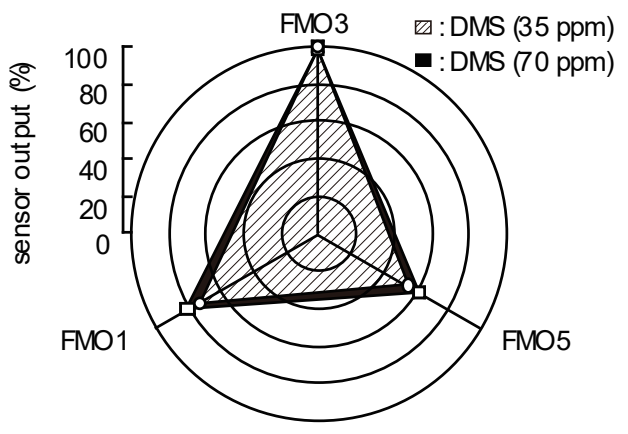

Fig. 7. Radar triangle chart of the biosniffer output with FMO1, 3, and 5 for dimethyl sulfide of 35 (hatched: front) and $70 \mathrm{ppm}$ (filled: back). The sensor outputs are nondimensionalized as a percentage (\%) of each device signal relative to the maximum output of the FMO3 sniffer. 
As Fig. 7 indicates, DMS (35 and $70 \mathrm{ppm}$ ) exhibits a typical triangle shape that is different from those of MM and TMA reported results. ${ }^{(8-10)}$ Those triangle patterns of the biosniffer with FMO1, 3 , and 5 were independent of their concentration, but identifies the species of the smell substance. The pattern recognition approach of using the specificity of xenobiotic-metabolizing enzymes such as FMO would improve the gas selectivity of the biosniffer. From those points of view, the opticalfiber sensor will be suitable for constructing the arrayed intelligent nose system for the assessment of multi-analyte vapor.

\section{Conclusions}

The optical biosniffer for DMS was constructed by immobilizing FMO onto the tip of a fiber optic oxygen sensor coated with an oxygen-sensitive ruthenium organic complex together with a reaction unit. The optical biosniffer with a cyclic reaction with ascorbic acid (10.0 mmol/l AsA) as the reducing reagent was used to measure DMS vapor with concentrations from 2.1 to $126 \mathrm{ppm}$ with gas selectivity based on the FMO substrate specificity. The gas selectivity could be improved by the pattern recognition approach with the isoform FMO signals. The sniffer was also used to detect gaseous DMS from a seaweed sample as a food application.

A potential application of the fiber sensor includes a smart nose system for the continuous monitoring of an odorous multi-analyte by suitably arraying the optical fiber. We will report on other optical biosniffers and the smart nose in the near future.

\section{Acknowledgements}

This work was supported by the Japan Society for the Promotion of Science (JSPS) Grant-inAid for Scientific Research System, Japan Science and Technology Agency (JST) and Ministry of Education, Culture, Sports, Science and Technology (MEXT) Special Funds for Education and Research “Advanced Research Program in Sensing Biology”.

\section{References}

1 International Programme on Chemical Safety: International Chemical Safety Cards (ICSC) 0878 dimethyl sulfide (1999).

2 C. T. Dolphin, A. Janmohamed, R. L. Smith, E. A. Shephard, and I. R. Phillips: Nat. Genet. 17 (1997) 491.

3 R. L. Haining, A. P. Hunter, A. J. M. Sadeque, R. M. Philpot, and A. E. Rettie: Drug Metab. Dispos. 25 (1997) 790.

4 D. H. Lang, C. K. Yeung, R. M. Peter, C. Ibarra, R. Gasser. K. Itagaki, R. M. Philpot, and A. E. Rettie: Biochem. Pharmacol. 56 (1998) 1005.

5 E. Toba and M. Ichikawa: Trans. IEEJ-C 112 (1992) 769 (in Japanese).

6 E. Toba, M. Ichikawa, A. Kazama, T. Nishimatsu, and H. Aizawa: Trans. IEEJ-E 119 (1999) 27 (in Japanese).

7 E. Toba: J. SICE 39 (2000) 253.

8 K. Mitsubayashi, M. Suzuki, E. Tamiya, and I. Karube: Anal. Chim. Acta 289 (1994) 27.

9 K. Mitsubayashi and Y. Hashimoto: IEEE Sens. J. 2 (2002) 133.

10 K. Mitsubayashi and Y. Hashimoto: Sens. Actuators, B 83 (2002) 35.

11 K. Ichimura: J. Polym. Sci. 22 (1984) 2817.

12 S. Uchiyama, H. Shimizu, and Y. Hasebe: Anal. Chem. 66 (1994) 1873.

13 Y. Hasebe, K. Oshima, O. Takise, and S. Uchiyama: Talanta 42 (1995) 2079.

14 Y. Hasebe: Chem. Sens. 14 (1998) 116. 